\title{
Maximizing the Potential of Patient-Reported Assessments by Using the Open-Source Concerto Platform With Computerized Adaptive Testing and Machine Learning
}

Conrad Harrison ${ }^{1}$, BSc, MBBS, MRCS; Bao Sheng Loe ${ }^{2}$, PhD; Przemysław Lis ${ }^{2}$, MSc; Chris Sidey-Gibbons ${ }^{3}$, PhD

${ }^{1}$ Nuffield Department of Orthopaedics, Rheumatology and Musculoskeletal Sciences, University of Oxford, Oxford, United Kingdom

${ }^{2}$ The Psychometrics Centre, University of Cambridge, Cambridge, United Kingdom

${ }^{3}$ MD Anderson Center for INSPiRED Cancer Care, University of Texas, Houston, TX, United States

Corresponding Author:

Conrad Harrison, BSc, MBBS, MRCS

Nuffield Department of Orthopaedics, Rheumatology and Musculoskeletal Sciences

University of Oxford

The Botnar Research Centre

Windmill Road

Oxford, OX3 7LD

United Kingdom

Phone: 441865227374

Email: conrad.harrison@medsci.ox.ac.uk

\begin{abstract}
Patient-reported assessments are transforming many facets of health care, but there is scope to modernize their delivery. Contemporary assessment techniques like computerized adaptive testing (CAT) and machine learning can be applied to patient-reported assessments to reduce burden on both patients and health care professionals; improve test accuracy; and provide individualized, actionable feedback. The Concerto platform is a highly adaptable, secure, and easy-to-use console that can harness the power of CAT and machine learning for developing and administering advanced patient-reported assessments. This paper introduces readers to contemporary assessment techniques and the Concerto platform. It reviews advances in the field of patient-reported assessment that have been driven by the Concerto platform and explains how to create an advanced, adaptive assessment, for free, with minimal prior experience with CAT or programming.
\end{abstract}

(J Med Internet Res 2020;22(10):e20950) doi: 10.2196/20950

\section{KEYWORDS}

computerized adaptive testing; computerized adaptive test; CAT; machine learning; patient reported outcome measures; outcome assessment; Concerto

\section{Introduction}

\section{Patient-Reported Assessment}

Patient-reported outcome measures (PROMs) measure the outcomes of health care that are most meaningful to patients. The importance and validity of a patient-centered approach to outcome assessment is gaining widespread acceptance across a diverse range of stakeholders including clinicians, researchers, clinical commissioners, health care business strategists, and patients themselves [1-4].

Despite growing interest in the use of patient-reported assessments, there are considerable barriers to their use, especially in time-pressured clinical environments. Two barriers, in particular, are both problematic and addressable. First, questionnaires can be burdensome to complete, especially when multiple domains of patient health are assessed at the same time [5]. Second, it can be unclear to health care professionals what actions should be taken based on the information received. In this paper, we will discuss strategies to reduce the burden of patient-reported assessment and improve the actionability and relevance of feedback. Specifically, it introduces modern psychometric theories, which can be used to create individualized assessments and reduce the burden of completion, as well as techniques for providing individualized feedback.

\section{Modern Psychometrics and Item-Response Theory}

The accuracy, reliability, and validity of patient-reported assessments are underpinned by complex psychometric 
statistical theory. Applying psychometric methods ensures that scores generated from PROMs can be used with high confidence in clinical practice and research [6].

Psychometrics can be divided into two broad domains. The first, referred to as classical test theory, uses correlational statistics to assess questionnaire properties, for example, how well the responses to certain items (questions) correlate with each other [7]. The second, known as modern test theory, uses probabilistic models to determine the properties of individual items [6].

The benefit of using modern test theory over classical test theory is that modern test theory allows researchers to evaluate the psychometric properties of individual items in relation to a targeted trait, whereas classical test theory correlational statistics mainly focuses on test-level performance. A more detailed comparison between the two approaches can be found elsewhere [6-8]. One of the biggest advantages of using modern test theory is that item properties can be used in a computerized adaptive testing (CAT) environment.

\section{CAT}

CAT refers to a process of selecting the most informative items for people responding to questionnaires. In contrast to fixed-length assessments, where a standard set of items are presented to every respondent all at once, CAT employs a psychometric algorithm to select items one at a time based on the amount of information they will provide about the individual assessment taker [9]. Each item is calibrated using statistical models described by modern test theory, and this process provides parameters for each item which are then used by the CAT algorithm to both calculate a respondent's score and select items [5].

After each response, CAT algorithms calculate a respondent's score based on the information available and select the next most informative item to administer. As more items are answered, the person's score is calculated with increasing accuracy. The CAT will eventually terminate when a stopping rule has been met. Stopping rules are typically based on a prespecified time limit, the number of items, the minimum standard error of measurement (SEM), or a combination thereof. Adapting assessments in this way can make them either briefer, more accurate, or in certain cases, both [10].

Many studies have assessed the impact of CAT on the length and accuracy of patient-reported outcome assessments. Experiments conducted both in silico and using human participants have robustly demonstrated that CAT can reduce the length of assessments by more than $50 \%$ while keeping excellent agreement between fixed-length assessment scores and CAT [5,11-14].

Despite their impressive performance, the uptake of currently available CAT platforms has been limited, including the Patient-Reported Outcome Measurement Information System (PROMIS) CAT which is accessible in the United States within the Epic electronic health record system [15]. In order to improve the uptake of this transformative technology and move toward truly patient-centered care, we must provide a more accessible way to implement CAT platforms in clinical practice and research. Research has demonstrated that PROM interventions are likely to have the greatest positive impact on patient outcomes when they are closely aligned with clinical care [16], and to achieve this we must administer such interventions through a versatile platform that meets the needs of clinicians, researchers, and patients.

\section{Machine Learning}

Machine learning refers to the process of developing or training algorithms to recognize patterns in existing data and to use this knowledge to make successful predictions with new data [17]. A great deal of enthusiasm has been shown for machine learning, as it has demonstrated exceptional performance in a variety of tasks including predicting the outcome of individuals following certain medical interventions, interpreting diagnostic images, and assessing the meaning of open-text passages [17-20].

There are a number of ways in which the collection and analysis of patient-reported data could be improved using machine learning. For instance, a branch of machine learning known as natural language processing may be used to generate quantifiable information from unstructured passages of open text [20]. Patient-reported assessments with integrated machine learning functions could include written (or spoken) patient responses, quantify said responses in a meaningful way, and use them to make recommendations for patient care or service improvement.

\section{Concerto}

Concerto was developed with the intention of providing a secure, versatile, and easy-to-use platform for creating patient-reported assessments that can incorporate CAT and machine learning. It is free to use and features a point-and-click interface that can be used to build advanced assessments with minimal prior programming experience [21].

Assessments created in Concerto are administered through highly adaptable front-end user interfaces that can be accessed from computers, smartphones, and electronic tablets. These interfaces are built similarly to websites, using HTML, JavaScript and CSS. The Concerto platform comes with inbuilt stock templates for users that do not wish to write their own code.

This user interface interacts with back-end functions, which can include scoring, CAT, and/or machine learning algorithms, using the $\mathrm{R}$ programming language. $\mathrm{R}$ programming has become popular among statisticians and data scientists for its breadth and accessibility [22]. There are currently over 15,000 available $\mathrm{R}$ packages, which can be used free of charge for statistical computing tasks including psychometric analyses, adaptive testing, and machine learning [23]. Concerto incorporates prewritten $\mathrm{R}$ code that can administer non-adaptive assessments or computerized adaptive tests, using item parameter tables that are uploaded by the user. The code is fully customizable for developers wishing to create more specialist assessments.

Patient data are stored securely using the MySQL database management system. The Concerto platform itself can be installed on Amazon cloud-based servers that comply with rigorous security demands. Alternatively, it can be installed on local servers (eg, those belonging to a health care provider) to comply with institutional security protocols. This has enabled 
the platform to be used successfully in clinical trials and for routine clinical care in the British National Health Service [24].

The Concerto platform can present assessment results immediately. Assessments can be presented in many forms depending on the needs of the end user. For example, radial plots can capture multiple dimensions of a person's health state (eg, different PROM subscale scores) at discrete times, and trend plots can show how a person's scores have changed over time or following major clinical events. Scores can be compared to normative values or other interpretability estimates, and SEMs can be presented alongside CAT scores. Respondents can even receive personalized written feedback to contextualize results (eg, "Your result is... This means..."). Providing immediate graphical and text-based feedback in this way has been shown to improve the experience of assessment when compared with traditional administration [2]. Results can be directly imported into a person's electronic health record through application programming interfaces.

In the following section, we demonstrate how a new Concerto user can create a computerized adaptive test for the Centre for Epidemiologic Studies Depression (CES-D) PROM.

\section{Concerto: a Worked Example}

\section{Installation}

Up to date installation guidance for personal Concerto use can be found at the Concerto GitHub webpage [25]. Readers should be aware that if they choose to use the Amazon Web Service (AWS) for installation, they will need to submit credit or debit card details as part of the registration process. Provided the default instance type (t2.micro) is selected, the following exercise should fall under Amazon's Free Tier. Readers are solely responsible for any costs they incur, and we would recommend that inexperienced AWS users take care when using the service.

\section{Download CES-D Items}

To complete this exercise, readers will need to download a CSV file that contains the item wordings, item response theory parameters and response options for the CES-D. This is available to download from the Open Science Framework [26].

We will refer to this table as a "flat" item table because all the data are stored in one layer (ie, there are no sub-tables within it).

Download the flat item table, install Concerto, and log in.

\section{Create a New Assessment}

On the Tests tab, click Add new.

Enter the name of your assessment in the Name box (eg, CESD_adaptive). The Type dropdown box should be set to flowchart. Click Save. Your test should appear under the Tests tab.

\section{Create a Table to Store Item Responses}

Click on the Data Tables tab and select Starter content. Click Edit next to the assessmentResponses table (see Figure 1). Click Copy and change the name of the table (eg, CESDResponses). Click Save. This action saves a new table in which to store your responses.

Figure 1. Concerto screenshot: creating a data table to store item responses.

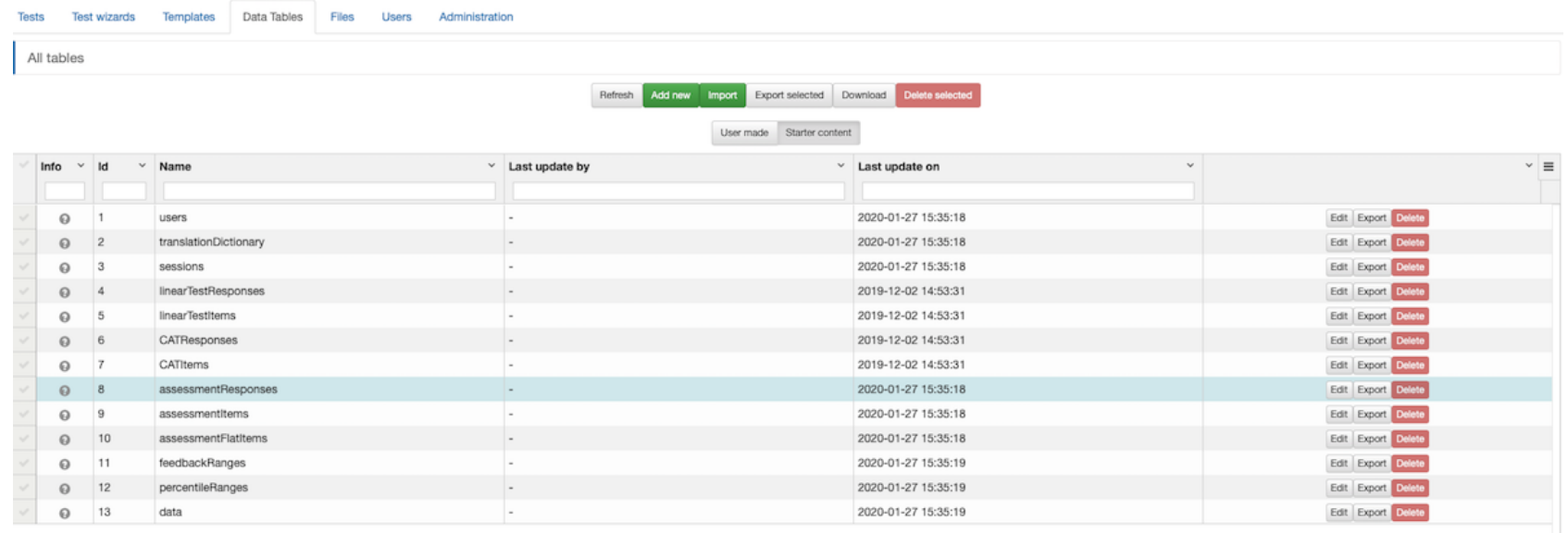

\section{Upload Your Items}

Click on All tables and select User made. Click Add new and type the name of your item table (eg, CESDFlatItems). Click Save. Your item table should appear in the list of user-made tables.
Click Edit next to your new item table. Click Upload CSV. Check the Restructure and Header row boxes. Use the Choose File button to select your flat item table and click Save. Alternatively, when the column header names in the CSV file are identical to those in the default flat item table, this can be copied over from the starter content in the same way as the item 
response table. The CSV file can then be uploaded with the Restructure box unchecked. This may improve system performance when collecting a large volume of responses by preserving certain database column types.

\section{Open the Flow Chart}

Under the Tests tab, click Edit next to your assessment. In the Test flow window, you will see your assessment displayed as a flow chart. It will have two nodes: test start and test end, which have a yellow output port and a white input port, respectively. Drag the test end node towards the right-hand side of the window to create some empty space between your nodes. Right-click the space between the nodes to create a third node (see Figure 2). Select assessment. The assessment node wizard should open automatically, with the Items tab preselected.

Figure 2. Concerto screenshot: opening the flowchart.
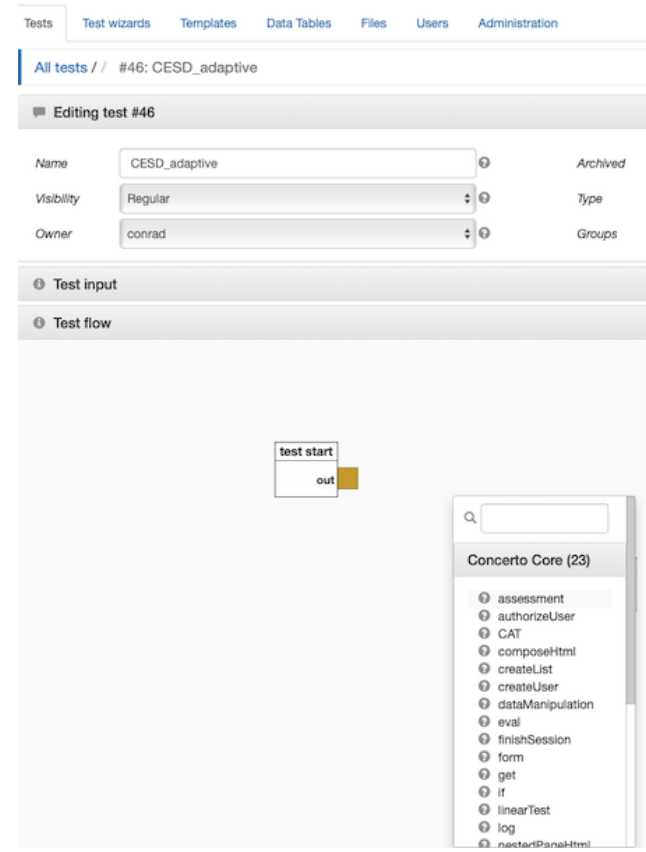

\section{Customize Your CAT}

Under Type, select Flat Table from the drop-down menu. Click the launch setter dialog icon under Flat Table. Select your item table from the Table drop-down menu and click Save. Select CAT from the Order drop-down menu.

Under the Stopping Rules tab, you can set stopping rules for your CAT. Try setting Minimum Accuracy to 0.5. This number represents the SEM that your assessment will achieve before terminating.

Under the CAT Options tab, select GRM from the Model drop-down menu. This relates to the psychometric model parameters which we will use for the CAT. In this example, our item parameters relate to a model known as the graded response model (GRM) [27].

Under the Responses tab, click the launch setter dialog icon next to Response Bank. Select your responses table from the Table drop-down menu. Click Save. Check the boxes next to Calculate Theta and Calculate SEM.

Under the Templates tab, enter a name for your assessment in the Title box (eg, CESD). Click the launch setter dialog icon under Instructions. Type some instructions for your assessment (eg, "Below is a list of the ways you might have felt or behaved - please tell me how often you have felt this way during the past week"). Click Save. Uncheck the Show Page Info box. Click Save.

\section{Add the CAT to Your Assessment}

Connect the test start node to the assessment node by dragging the yellow output port on the test start node to the white input port on the assessment node. Click the red plus sign on the assessment node to create new return ports. Check the boxes next to theta and sem and click Save. This enables the assessment node to pass on a person's score (theta) and the SEM associated with that score.

Right click the empty space between your assessment node and test end node to create a fourth node. Select scoring. Choose Percentile (normal distribution) from the Score Type drop-down menu. Enter a mean of 0 and a standard deviation of 1 . Click Save.

On the scoring node click the blue plus sign to add an input port. Check the box next to rawScore and click Save. Click the red plus sign on the scoring node to add a return port. Check the box next to score and click Save. Connect the yellow output port from the assessment node to the white input port of the scoring node. Connect the theta return port to the rawScore input port.

\section{Create a Results Page With Contextual Feedback}

Right click the empty space between your scoring node and your test end node to create a fifth node; you may need to reposition the nodes to make sufficient space. Select showPage. Enter a title (eg, CESD), then click the launch setter dialog icon under Content and copy the following: 
Theta is $\{\{$ theta $\}\}$ SEM is $\{\{\operatorname{sem}\}\}$.

Your score is higher than $\{\{$ percent $\}\} \%$ of the general population.

The double braces (curly brackets) specify which values for our feedback page to take from our scoring node. Click Save. Clear the Button Label and click Save again.

Click the blue plus sign on the showPage node to create a new input port. Type theta into the text box, noting the lowercase. Click Add.

Click the blue plus sign on the showPage node to create a second input port. Type sem into the text box, again using lowercase. Click Add.

Figure 3. Concerto screenshot: connecting nodes.

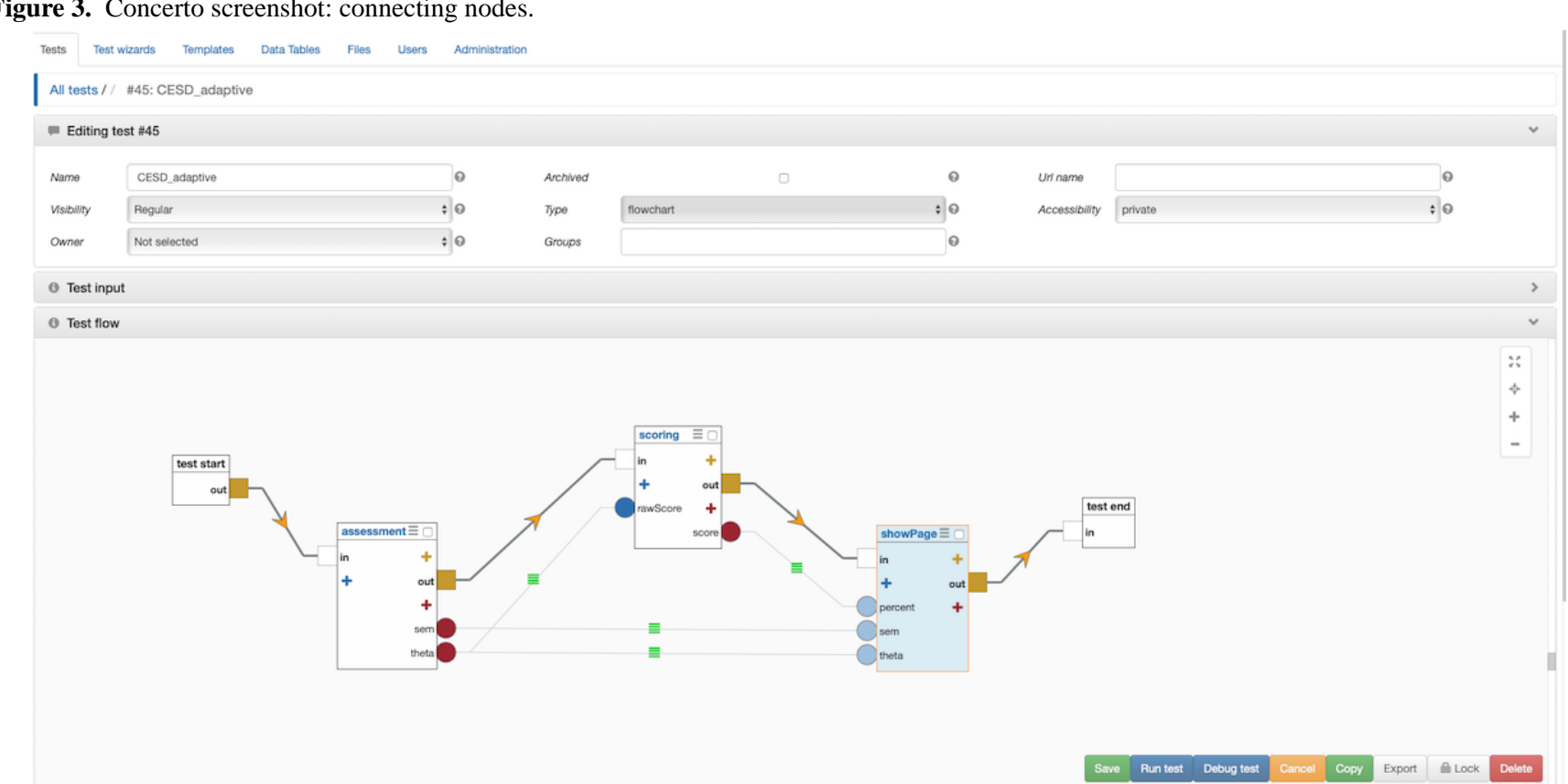

\section{Concerto Case Studies}

\section{Quality of Life Assessment}

The World Health Organization Quality of Life (WHOQOL)-100 questionnaire was developed in the 1990s by the WHOQOL group as part of an international collaborative effort to produce a generic, cross-cultural, and widely accepted tool for measuring quality of life. The 100-item questionnaire assesses quality of life across six domains (physical, psychological, social, emotional, independence and spiritual), and is also available as a 26 item (WHOQOL-BREF) version with four domains (physical, psychological, social and environmental) [28].

In 2016, our team calibrated item banks from the 100-item questionnaire using modern test theory and trialed a unidimensional CAT version of the questionnaire using simulated data across four domains (physical, psychological, social and environmental). The CAT version of the questionnaire
Click the blue plus sign on the showPage node to create a third input port. Type percent into the text box, again using lowercase. Click Add.

Connect the sem return port on the assessment node to the sem input port on the showPage node.

Connect the theta return port on the assessment node to the theta input port on the showPage node.

Connect the score return port on the scoring node to the percent input port on the showPage node.

Connect the yellow output port on the scoring node to the white input port on the showPage node. Connect the yellow output port on the showPage node to the white input port on the test end node (see Figure 3). Click Save. You are now ready to run your assessment. Responses will be stored in the CESDResponses table. used $43 \%$ and $75 \%$ fewer items than the WHOQOL-BREF and WHOQOL-100 questionnaires respectively, at comparable levels of reliability [5]. The WHOQOL CAT, when administered through Concerto, takes a mean of 121 seconds to complete, approximately 10 minutes faster than the WHOQOL-100 [29].

\section{Analyzing Open-Text Feedback of Doctors' Performance With Machine Learning}

Multisource feedback has become a routine part of UK doctors' training and appraisal. Often, these feedback assessments contain open-text comments from colleagues about a doctor's performance [30]. Automating the analysis of these comments could provide real-time, objective insights into both an individual doctor's performance and the interpersonal dynamics of a team or department.

In 2017, our team demonstrated the ability for machine learning algorithms to classify open-text feedback from the General Medical Council Colleague Questionnaire (GMC-CQ) into five themes, with human-level accuracy. These themes were 
innovation, interpersonal skills, popularity, professionalism and respect. Doctors classified as professional, respected or with good interpersonal skills achieved higher GMC-CQ scores than those who were not classified as such [31].

Interested readers can freely apply these algorithms to their own open text comments using the Concerto-based platform [32].

\section{Improving Assessments of Patient Experience With Machine Learning}

Patient-reported experience measures (PREMs) that take the form of questionnaires are often limited by a ceiling effect. This describes a skew towards positive reporting, which can limit the discriminative ability of a PREM and mask poor service performance [33,34].

We have shown that in the context of UK primary care, spoken feedback from patients can provide more accurate, more detailed, and more actionable insights into the consultation experience than questionnaire results alone. In one study, we found a tendency for patients to rate consultation experiences positively when answering items from the interpersonal skills domain of the national GP Patient Survey, although nearly $60 \%$ of respondents who rated their consultation as "good" provided contradictory feedback when interviewed about their experience [35].

Using Concerto, we have developed a patient satisfaction assessment called INSPiRES (Innovative Systems for Patient Reported Experience in Surgery) that combines multiple choice responses with open-text analysis. During the assessment, respondents first select 1 of 12 emotions that describe how they feel about the care they have received. Next, respondents provide an open-text description indicating why they feel that way. Finally, respondents explain which part of their experience led to that feeling (eg, waiting times, cleanliness, care providers) by either selecting a prespecified option or entering free text. The tool is being trialed at the Brigham and Women's Hospital, Boston, MA, USA, and is expected to provide specific, actionable feedback that will drive service improvement [36]. Although currently used during surgical outpatient clinics, the assessment is also available as a smartphone app that patients can complete from home.

\section{Discussion}

In this article, we have introduced Concerto and demonstrated how to create an advanced, adaptive assessment, for free, with minimal prior experience of CAT or programming. Concerto assessments can incorporate other features, including those that use machine learning, although this is less straightforward at present. The platform has been used internationally to improve the performance of PROMs in research and clinical practice, to classify open-text assessments of health care providers, and to provide meaningful insights into the experience of health care delivery [2,24,31,32,36].

In future, Concerto could be used to develop and deploy advanced clinical decision support systems (CDSSs) that harness the power of CAT and machine learning to assist clinicians in making evidence-based decisions during daily practice. These systems, which can use patient-reported assessments to predict the outcomes of an individual following a health care intervention, are already being trialed to streamline UK GP referrals and support shared decision making in surgery [37]. Existing CDSSs, most notably the NHS Pathways CDSS, which is used by NHS 111 to triage over 14 million telephone calls a year [38], could be trained to automatically interpret spoken word or open-text through natural language processing.

Concerto-based assessments can be deployed on mobile devices as a tool for remote symptom monitoring. Besides the survival advantage this can bring patients with cancer [16], it has quite obvious implications for a broad range of domiciliary disciplines (eg, out-of-hospital palliative care, general practice, and psychiatry).

Patient-reported assessments can transform clinical practice, research, commissioning, and health care management strategies by measuring the impact of an intervention from the patient's perspective. To deliver the full potential of these assessments, they should be short, accurate, and acceptable to both respondents and those administering the assessment. They should be personalized, ask only the most relevant questions to an individual, and not be limited to multiple-choice responses. Results should be analyzed in real-time and presented to assessment users in an engaging and meaningful way. Where appropriate, data should be easily available for use in secondary analyses including predictive models. These assessments must integrate easily with health care services, including interoperating with electronic health records. Patient data must be stored and processed securely and ethically.

The Concerto platform bridges the implementation gap between the assessments of today and those of tomorrow.

\section{Conflicts of Interest}

BSL, PS, and CS-GS are employed by the Psychometrics Centre, University of Cambridge. The Concerto platform is open source and free to use, but in some circumstances the Psychometrics Centre will receive revenue from organizations requiring technical support with the platform.

\section{References}

1. Weldring T, Smith SMS. Patient-Reported Outcomes (PROs) and Patient-Reported Outcome Measures (PROMs). Health Serv Insights 2013 Aug;6:61-68 [FREE Full text] [doi: 10.4137/HSI.S11093] [Medline: 25114561]

2. Geerards D, Pusic A, Hoogbergen M, van der Hulst R, Sidey-Gibbons C. Computerized Quality of Life Assessment: A Randomized Experiment to Determine the Impact of Individualized Feedback on Assessment Experience. J Med Internet Res 2019 Jul 11;21(7):e12212. [doi: 10.2196/12212] 
3. Porter ME. What Is Value in Health Care? N Engl J Med 2010 Dec 23;363(26):2477-2481. [doi: 10.1056/NEJMp1011024]

4. Black N. Patient reported outcome measures could help transform healthcare. BMJ 2013 Jan 28;346(jan28 1):f167-f167. [doi: 10.1136/bmj.f167]

5. Gibbons C, Bower P, Lovell K, Valderas J, Skevington S. Electronic Quality of Life Assessment Using Computer-Adaptive Testing. J Med Internet Res 2016 Sep 30;18(9):e240. [doi: 10.2196/jmir.6053]

6. Nguyen TH, Han H, Kim MT, Chan KS. An Introduction to Item Response Theory for Patient-Reported Outcome Measurement. Patient 2014 Jan 9;7(1):23-35. [doi: 10.1007/s40271-013-0041-0]

7. DeVellis RF. Classical test theory. Med Care 2006 Nov;44(11 Suppl 3):S50-S59. [doi: 10.1097/01.mlr.0000245426.10853.30] [Medline: 17060836$]$

8. Cai L, Choi K, Hansen M, Harrell L. Item Response Theory. Annu. Rev. Stat. Appl 2016 Jun;3(1):297-321. [doi: 10.1146/annurev-statistics-041715-033702]

9. Weiss DJ, Vale CD. Adaptive Testing. Applied Psychology 1987 Jul;36(3-4):249-262. [doi: 10.1111/j.1464-0597.1987.tb01190.x]

10. Loe BS, Stillwell D, Gibbons C. Computerized Adaptive Testing Provides Reliable and Efficient Depression Measurement Using the CES-D Scale. J Med Internet Res 2017 Dec 20;19(9):e302 [FREE Full text] [doi: 10.2196/jmir.7453] [Medline: 28931496]

11. Young-Afat D, Gibbons C, Klassen A, Vickers A, Cano S, Pusic A. Introducing BREAST-Q Computerized Adaptive Testing: Short and Individualized Patient-Reported Outcome Assessment following Reconstructive Breast Surgery. In: Plastic and Reconstructive Surgery; 2019.

12. Geerards D, Klassen AF, Hoogbergen MM, van der Hulst RR, van den Berg L, Pusic AL, et al. Streamlining the Assessment of Patient-Reported Outcomes in Weight Loss and Body Contouring Patients. Plastic and Reconstructive Surgery 2019;143(5):946e-955e. [doi: 10.1097/prs.0000000000005587]

13. Harrison CJ, Geerards D, Ottenhof MJ, Klassen AF, Riff KWW, Swan MC, et al. Computerised adaptive testing accurately predicts CLEFT-Q scores by selecting fewer, more patient-focused questions. 2019 Nov;72(11):1819-1824. [doi: 10.1016/j.bjps.2019.05.039]

14. Bass M, Morris S, Neapolitan R. Utilizing Multidimensional Computer Adaptive Testing to Mitigate Burden With Patient Reported Outcomes. 2015 Nov Presented at: AMIA. Annu Symp proceedings AMIA Symp; 2015; San Fransico.

15. Cella D, Yount S, Rothrock N, Gershon R, Cook K, Reeve B, et al. The Patient-Reported Outcomes Measurement Information System (PROMIS). Medical Care 2007;45(Suppl 1):S3-S11. [doi: 10.1097/01.mlr.0000258615.42478.55]

16. Denis F, Basch E, Septans A, Bennouna J, Urban T, Dueck AC, et al. Two-Year Survival Comparing Web-Based Symptom Monitoring vs Routine Surveillance Following Treatment for Lung Cancer. JAMA 2019 Jan 22;321(3):306. [doi: 10.1001/jama.2018.18085]

17. Sidey-Gibbons JAM, Sidey-Gibbons CJ. Machine learning in medicine: a practical introduction. BMC Med Res Methodol 2019 Mar 19;19(1):64 [FREE Full text] [doi: 10.1186/s12874-019-0681-4] [Medline: 30890124]

18. Senders JT, Staples PC, Karhade AV, Zaki MM, Gormley WB, Broekman ML, et al. Machine Learning and Neurosurgical Outcome Prediction: A Systematic Review. World Neurosurgery 2018 Jan;109:476-486.e1. [doi: 10.1016/j.wneu.2017.09.149]

19. Esteva A, Kuprel B, Novoa RA, Ko J, Swetter SM, Blau HM, et al. Dermatologist-level classification of skin cancer with deep neural networks. Nature 2017 Jan 25;542(7639):115-118. [doi: 10.1038/nature21056]

20. Collobert R, Weston J, Bottou L, Karlen M, Kavukcuoglu K, Kuksa P. Natural language processing (almost) from scratch. J Mach Learn Res 2011:2493-2537.

21. The Psychometrics Centre. Concerto Adaptive Testing Platform. University of Cambridge. URL: https://www. psychometrics.cam.ac.uk/newconcerto [accessed 2020-10-02]

22. R: A language and environment for statistical computing. R Project. URL: http://www.r-project.org/ [accessed 2020-10-02]

23. Contributed Packages. CRAN. URL: https://cran.r-project.org/web/packages/ [accessed 2020-10-02]

24. Gibbons C. Introducing Concerto, an open-source platform designed to realise the potential of modern measurement theories. Eur Heal Psychol. (S) 2016;18:421.

25. Lis P. Concerto platform quickstart guide. GitHub. 2019. URL: https://github.com/campsych/concerto-platform/wiki/ quickstart-guide [accessed 2020-10-02]

26. Delivering the full potential of patient-reported assessments: computerized adaptive testing and machine learning using the open source Concerto platform. OSFHOME. URL: https://osf.io/8jypd/ [accessed 2020-10-14]

27. Samejima F. Erratum Estimation of latent ability using a response pattern of graded scores. Psychometrika 1970 Mar 1;35(1):139-139. [doi: 10.1007/bf02290599]

28. The Whoqol Group. Development of the World Health Organization WHOQOL-BREF Quality of Life Assessment. Psychol. Med 1998 May 01;28(3):551-558. [doi: 10.1017/S0033291798006667]

29. Velikova G, Valderas JM, Potter C, et al. Advances in Patient Reported Outcomes Research. In: Health Qual Life Outcomes. 2017 Oct 05 Presented at: Proceedings of Patient Reported Outcome Measure's (PROMs) Conference; 2017; Oxford p. 185 URL: https://hqlo.biomedcentral.com/articles/10.1186/s12955-017-0757-y [doi: 10.1186/s12955-017-0757-y] 
30. Whitehouse A, Hassell A, Wood L, Wall D, Walzman M, Campbell I. Development and reliability testing of TAB a form for $360^{\circ}$ assessment of Senior House Officers' professional behaviour, as specified by the General Medical Council. Medical Teacher 2009 Jul 03;27(3):252-258. [doi: 10.1080/01421590500047062]

31. Gibbons C, Richards S, Valderas JM, Campbell J. Supervised Machine Learning Algorithms Can Classify Open-Text Feedback of Doctor Performance With Human-Level Accuracy. J Med Internet Res 2017 Mar 15;19(3):e65. [doi: 10.2196/jmir.6533]

32. The Psychometrics Centre. Colleague feedback tool. Open Text Analysis. 2017. URL: www.OpenTextAnalysis.com [accessed 2020-10-02]

33. Hodson M, Andrew S, Michael Roberts C. Towards an understanding of PREMS and PROMS in COPD. Breathe 2013 Sep 01;9(5):358-364. [doi: 10.1183/20734735.006813]

34. GP Patient Survey 2015. NHS England. 2016. URL: https://www.england.nhs.uk/statistics/2016/01/07/gp-patient-survey-2015/ [accessed 2020-10-02]

35. Burt J, Newbould J, Abel G, Elliott MN, Beckwith J, Llanwarne N, et al. Investigating the meaning of 'good' or 'very good' patient evaluations of care in English general practice: a mixed methods study. BMJ Open 2017 Mar 02;7(3):e014718. [doi: 10.1136/bmjopen-2016-014718]

36. Cambridge Network. Converting clicks into care. Cambridge Network. URL: https://www.cambridgenetwork.co.uk/news/ $\underline{600685}$ [accessed 2020-10-02]

37. Price A, Smith J, Dakin H, et al. The Arthroplasty Candidacy Help Engine tool to select candidates for hip and knee replacement surgery: development and economic modelling. Health Technol Assess 2019 Jun;23(32):1-216. [doi: 10.3310/hta23320]

38. NHS Pathways. NHS Digital. URL: https://digital.nhs.uk/services/nhs-pathways [accessed 2020-04-16]

\author{
Abbreviations \\ CAT: computerized adaptive test \\ CDSS: clinical decision support system \\ CES-D: Centre for Epidemiologic Studies Depression Scale \\ GMC-CQ: General Medical Council colleague questionnaire \\ GRM: graded response model \\ INSPiRES: Innovative Systems for Patient Reported Experience in Surgery \\ NLP: natural language processing \\ PREM: patient-reported experience measure \\ PRO: patient reported outcome \\ PROM: patient-reported outcome measure \\ PROMIS: Patient-Reported Outcome Measurement Information System \\ SEM: standard error of measurement \\ WHOQOL: World Health Organization quality of life
}

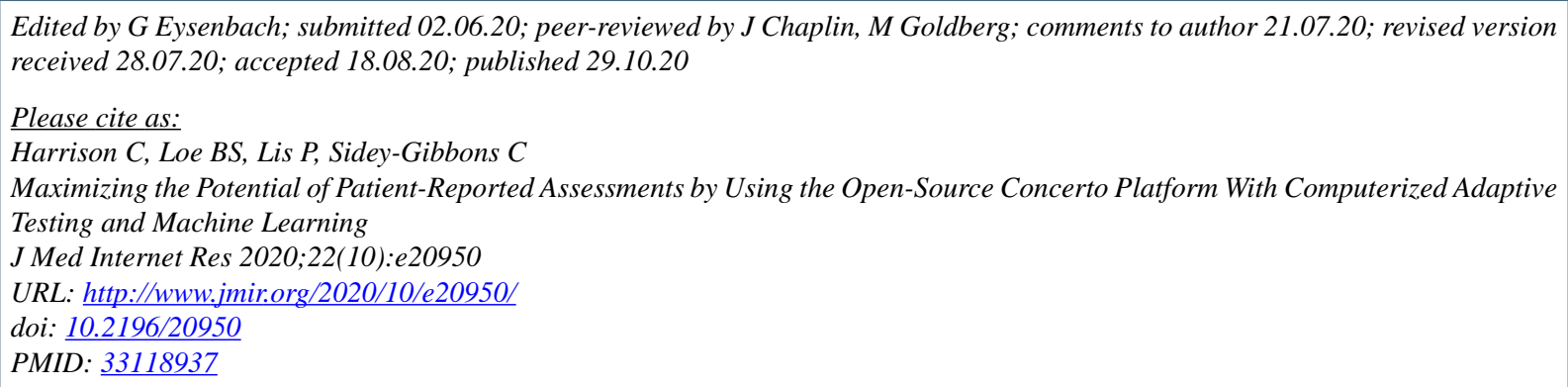

(C) Conrad Harrison, Bao Sheng Loe, Przemysław Lis, Chris Sidey-Gibbons. Originally published in the Journal of Medical Internet Research (http://www.jmir.org), 29.10.2020. This is an open-access article distributed under the terms of the Creative Commons Attribution License (https://creativecommons.org/licenses/by/4.0/), which permits unrestricted use, distribution, and reproduction in any medium, provided the original work, first published in the Journal of Medical Internet Research, is properly cited. The complete bibliographic information, a link to the original publication on http://www.jmir.org/, as well as this copyright and license information must be included. 